\title{
Evidence that full-mouth scaling superior to conventional treatment approaches is unclear
}

\author{
Abstracted from \\ Eberhard J, Jepsen S, Jervøe-Storm PM, Needleman I, Worthington HV. \\ Full-mouth treatment modalities (within 24 hours) for chronic periodontitis in adults. \\ Cochrane Database Syst Rev 2015; 4: Art. No. CD004622. DOI: 10.1002/14651858.CD004622.pub3. \\ Address for correspondence: Luisa Fernandez Mauleffinch, Managing Editor, Cochrane Oral Health Group, \\ School of Dentistry, The University of Manchester, JR Moore Building, Oxford Road, Manchester, M13 9PL, UK. \\ E-mail: luisa.fernandez@manchester.ac.uk
}

\section{Question: Should adults with chronic periodontitis have their treatment carried out within a 24-hour period?}

Data sources The Cochrane Oral Health Group Trials Register, the Cochrane Central Register of Controlled Trials, Medline, Embase, CINHAL, the US National Institutes of Health Trials Register and the WHO Clinical Trials Registry Platform.

Study selection Randomised controlled trials (RCTs) with at least three months follow-up comparing full-mouth scaling and root planing within 24 hours with adjunctive use of an antiseptic such as chlorhexidine (FMD) or without the use of antiseptic (FMS), compared to conventional quadrant scaling and root planing (control).

Data extraction and synthesis Methodological guidelines of the Cochrane Collaboration were followed. Two review authors selected the papers and determined eligibility based on the titles and abstracts, and any disagreements were resolved by discussion. Four review authors carried out the data extraction. The characteristics of studies excluded at the full-text stage are described in detail. The Cochrane risk of bias tool was used to assess bias in the included studies. Tooth loss and changes in pocket probing depths were the primary outcome measures while attachment level and bleeding on probing were the secondary outcomes. Results Twelve trials recruiting 389 participants were included. No studies assessed the primary outcome tooth loss. Ten trials compared FMS and control and there was no evidence for benefit of FMS in any of the outcome measures. Six trials were included in the meta-analysis comparing FMD and control, which did not reveal a benefit for FMD over conventional scaling and root planing. Three trials compared FMS and FMD and did not demonstrate a benefit of one over the other. The quality of evidence was assessed as 'low' due to study design limitations and the small number of trials and participants.

Conclusions From the 12 included trials there is no clear evidence that FMS or FMD provide additional benefit compared to conventional scaling and root planing. In practice, the decision to select one approach to non-surgical periodontal therapy over another should include patient preference and the convenience of the treatment schedule.

This paper is based on a Cochrane Review published in the Cochrane Library 2015, issue 4 (see www.thecochranelibrary.com for information). Cochrane Reviews are regularly updated as new evidence emerges and in response to feedback, and the Cochrane Library should be consulted for the most recent version of the review.

\section{Commentary}

It has long been established that plaque is the primary aetiological factor in periodontal disease. ${ }^{1}$ It is estimated that $47 \%$ of people have periodontitis. ${ }^{2}$ Treatment of periodontal disease aims to reduce the bacterial load and involves oral hygiene instruction with supraand sub-gingival debridement of the crown and roots of teeth. ${ }^{3}$ This is usually carried out over multiple sessions spaced one to two weeks apart. There is a wealth of evidence to support the effectiveness of this approach ${ }^{4-6}$ and it remains the most popular approach today. The idea that periodontal pathogens from un-instrumented sites can re-colonise recently instrumented pockets brought about the concept of full-mouth treatment within 24 hours. $^{7}$ Since then, there has been conflicting evidence produced about the efficacy of these full-mouth treatments over conventional scaling and root planing. Lang et al. produced a systematic review and metaanalysis following the $6^{\text {th }}$ European Workshop in Periodontology. They found FMD and FMS had statistically significant benefits over conventional SRP in some pocket depth reductions and clinical attachment gains; however, these findings were inconsistent and were too small to be considered clinically relevant. They concluded all three treatment approaches could be recommended, as did two other reviews, published around the same time. ${ }^{9,10}$ In contrast, Teughels et al. ${ }^{11}$ published a review which concluded one-stage full-mouth treatment approaches provide a significant clinical and microbiological improvement without any disadvantages or risks to the patient. However, this review did not provide any statistical analysis and was published by the same group from Leuven, Belgium, who produced the original studies and investigated onestage full-mouth treatment modalities.

The aim of this Cochrane review was to update a previous review on this topic from $2008,{ }^{12}$ and to ensure the evidence for this question is up to date. This updated review includes an additional five studies on top of the seven originally included in 2008, giving a total of 12 RCTs for analysis. This review, being from the Cochrane Collaboration, is exhaustive in its nature. Their rigid methodological approaches to these reviews allow only studies that meet strict inclusion criteria to be included. They also include a detailed description of studies that were excluded at the full-text stage, which not all systematic reviewers do. The evidence suggests that while full-mouth treatment modalities are effective in the treatment of chronic periodontitis they provide no additional benefit over conventional scaling and root planing. This update does not change the conclusions of the original review. The overall quality of evidence was assessed as being 'low' due to design 
limitations leading to a risk of bias, and the small number trials and participants.

The applicability of these results to clinical everyday practice could be judged to be unreliable due to the fact that all the trials were conducted in university dental departments. The authors concluded that the decision to select one approach over another should include patient preference and convenience of the schedule. However, according to Teughels et al. ${ }^{11}$ there was better compliance and acceptability with the one-stage full-mouth approach, benefiting both the clinician and patient.

Conventional scaling and root planing remains the most proven treatment approach for chronic periodontitis and against which other treatment modalities will continue to be measured. Whether more research can establish if full-mouth treatment modalities can replace this traditional approach to treatment remains to be seen.

The authors of this review advise that further research would be beneficial, given the quality of evidence was assessed as low, however, there may be difficulty in achieving this. More trials with a larger number of participants are required. Longer followup periods are required to assess tooth loss (this being a primary outcome measure). However, the fact that supportive periodontal therapy begins soon after initial treatment means determining the long-term effects of the initial treatment modality may be challenging.

Some of the issues, which contributed to the high risk of bias in this review, may be simpler to correct. In this review seven trials did not report adequate information about random assignment, and three did not carry this out. Ensuring the participants are randomly assigned to a treatment modality is important to provide allocation concealment and future research should endeavour to do this. Eberhard also suggests that further research should focus on the financial implications and patient burden of these modalities.

Mark McLaughlin and Brett Duane Dublin Dental University Hospital, Dublin, Ireland

1. Loe H, Theilade E, Jensen SB. Experimental Gingivitis in Man. J Periodontol 1965; 36: 177-187.

2. Eke PI, Dye BA, Wei L, et al. Update on Prevalence of Periodontitis in Adults in the United States: NHANES 2009 to 2012. / Periodontol 2015; 86: 611-622.

3. Claffey N, Polyzois I, Ziaka P. An overview of nonsurgical and surgical therapy. Periodontol 2000. 2004; 36: 35-44.

4. Badersten A, Nilveus R, Egelberg J. Effect of nonsurgical periodontal therapy. II. Severely advanced periodontitis. / Clin Periodontol 1984; 11: 63-76.

5. Heitz-Mayfield LI, Trombelli L, Heitz F, Needleman I, Moles D. A systematic review of the effect of surgical debridement vs non-surgical debridement for the treatment of chronic periodontitis. J Clin Periodontol 2002; 29: 92-102.

6. Van der Weijden GA, Timmerman MF. A systematic review on the clinical efficacy of subgingival debridement in the treatment of chronic periodontitis. / Clin Periodontol 2002; 29: 55-71.

7. Quirynen M, Bollen CM, Vandekerckhove BN, Dekeyser C, Papaioannou W, Eyssen H. Full-vs. partial-mouth disinfection in the treatment of periodontal infections: shortterm clinical and microbiological observations. / Dent Res 1995; 74: 1459-1467.

8. Lang NP, Tan WC, Krahenmann MA, Zwahlen M. A systematic review of the effects of full-mouth debridement with and without antiseptics in patients with chronic periodontitis. / Clin Periodontol 2008; 35: 8-21.

9. Farman M, Joshi RI. Full-mouth treatment versus quadrant root surface debridement in the treatment of chronic periodontitis: a systematic review. Br Dent / 2008; 205: E18; discussion 496-497.

10. Tomasi C, Wennström JL. Full-mouth treatment vs. the conventional staged approach for periodontal infection control. Periodontol 2000. 2009; 51: 45-62.

11. Teughels W, Dekeyser C, Van Essche M, Quirynen M. One-stage, full-mouth disinfection: fiction or reality? Periodontol 2000. 2009; 50: 39-51.

12. Eberhard J, Jepsen S, Jervøe-Storm PM, Needleman I, Worthington HV. Full-mouth disinfection for the treatment of adult chronic periodontitis. Cochrane Database Syst Rev 2008; (1): CD004622.

Evidence-Based Dentistry (2016) 17, 23-24. doi:10.1038/sj.ebd.6401154 